\title{
Implementing Dialectical Behavior Therapy Skills Training for Emotional Problem Solving for Adolescents (DBT STEPS-A) in a Low-Income School
}

\author{
Carla D. Chugani ${ }^{1}$ (1) . Courtney E. Murphy ${ }^{1} \cdot$ Janine Talis $^{1} \cdot$ Elizabeth Miller $^{1} \cdot$ Christopher McAneny $^{2}$. \\ Daniel Condosta ${ }^{3} \cdot$ Julie Kamnikar $^{3} \cdot$ Edward Wehrer $^{4} \cdot$ James J. Mazza $^{5}$
}

Accepted: 22 July 2021 / Published online: 5 August 2021

(c) The Author(s), under exclusive licence to Springer Science+Business Media, LLC, part of Springer Nature 2021

\begin{abstract}
Adolescents living in low-income areas often have high need for mental health supports due to experiences of poverty and trauma, coupled with limited access and availability of such supports. This study investigated the implementation of a socio-emotional learning curriculum titled, "Dialectical Behavior Therapy Skills Training for Emotional Problem Solving for Adolescents (DBT STEPS-A)," which was integrated into health classes in a low-income high school. While preliminary evidence suggests that DBT STEPS-A can be effective in reducing mental health symptoms in high school students, this study is the first to explore the program's acceptability, appropriateness, and feasibility when implemented in a low-income school. The implementation presented here also diverged from recommended training protocols due to time and cost limitations. Quantitative and qualitative data were collected from 29 school stakeholders prior to implementation and from 23 school stakeholders post-implementation. Our results indicate that DBT STEPS-A is acceptable and feasible for teachers involved in offering the program and that more work is needed to address appropriateness of the content for racially and socio-economically diverse students, ease of implementing lessons, and support for teachers using DBT STEPS-A skills outside of class. We conclude with a discussion of key implementation challenges and solutions generated.
\end{abstract}

Keywords Socio-emotional learning $\cdot$ Dialectical behavior therapy $\cdot$ Low-income schools $\cdot$ Implementation

\section{Literature Review}

Adolescents living in communities with low socioeconomic status (SES) have higher needs for mental health support due to experiences of discrimination, violence exposure,

Carla D. Chugani

carla.chugani@chp.edu

Courtney E. Murphy

ceb130@pitt.edu

Janine Talis

Janine.talis2@chp.edu

Elizabeth Miller

Elizabeth.miller@chp.edu

Christopher McAneny

cmcaneny@homelessfund.org

Daniel Condosta

dcondosta@steelvalleysd.org

Julie Kamnikar

jkamnikar@steelvalleysd.org and poverty (Shim et al., 2018; World Health Organization $\&$ Calouste Gulbenkian Foundation, 2014). Those attending low-income schools have higher rates of depression, anxiety, and engagement in violence (Coley et al., 2018), and those who qualify for free meals are significantly more

Edward Wehrer

ewehrer@steelvalleysd.org

James J. Mazza

mazza@uw.edu

1 Department of Pediatrics, Division of Adolescent and Young Adult Medicine, University of Pittsburgh School of Medicine, Pittsburgh, PA, USA

2 Homeless Children's Education Fund, Pittsburgh, PA, USA

3 Steel Valley High School, Munhall, PA, USA

4 Steel Valley School District, Munhall, PA, USA

5 University of Washington College of Education, Seattle, WA, USA 
likely to have mental health, social, and behavioral challenges (Deighton et al., 2019). Coupled with increased mental health needs, adolescents in low-SES communities have decreased access to mental health care arising from a number of factors, including stigma, long wait times, lack of providers specializing in mental health care for teens (Silberholz et al., 2017), limited finances/insurance coverage, and transportation.

School-based socio-emotional learning (SEL) programs are a highly promising strategy for universal access to mental health supports, yet they typically achieve small effects or mixed effects for mental health outcomes (National Academies of Sciences, 2019). Three recent meta-analyses have been conducted which examine the effects of SEL programs for a variety of standard SEL outcomes including skill building, academic achievement, behavior problems, and mental health (Durlak et al., 2011; Sklad et al., 2012; Taylor et al., 2017). These studies have consistently found that SEL programs achieve small effect sizes for mental health outcomes $(d=0.16-0.24)$. These small effects and mixed results may result from challenges in implementing such programs in the school system and issues with program fidelity. While quality implementation supports program effectiveness (Durlak et al., 2011), the implementation research-related to SEL is limited and focused on sub-constructs of overall implementation success such as fidelity or dosage (Low et al., 2016). More broadly, barriers to implementation of school-based mental health programs include competing responsibilities, logistical barriers, and lack of support from school administrators and teachers, whereas facilitators include having a social network among implementers, more organizational structure, and administrative support (Langley et al., 2010).

It is also noteworthy to mention that SEL programs may focus on many different types of skill building including areas such as goal setting, problem solving, or in the case of suicide prevention, help-seeking, and gatekeeper training designed to help individuals learn to recognize and respond to a person who is displaying warning signs of suicidality. SEL programs that aim to improve mental health may achieve small effects if the skills taught do not directly assist youth in learning how to cope, regulate emotions, or modify factors causing emotional distress. In many cases, these programs may actually be targeting mental well-being rather than emotional distress, which most rigorously evaluated SEL programs have been shown to have either low or no efficacy for reducing (Collaborative for Academic Social and Emotional Learning (CASEL), 2015). Even among SEL programs designed to prevent suicide, there is a lack of focus on teaching specific skills for reducing emotional arousal and distress. For example, the peer-delivered Sources of Strength program (LoMurray, 2005) is designed to promote identification and engagement with "trusted adults," encourages students who identify a friend as suicidal to seek out help, and encourages identification of coping resources (Wyman et al., 2010). Sources of Strength has been shown to be effective for increasing acceptability of help-seeking and perceived adult support for suicidality (Wyman et al., 2010). The Signs of Suicide program (MindWise Innovations, 2021), which has been shown to be effective for reducing suicide attempts in high school students, is designed to educate students about depression and suicide ideation, encourage intervention with suicidal peers, and promote help-seeking among youth experiencing suicide ideation (Schilling et al., 2016). Although SEL programs can achieve a range of important outcomes related to mental health, a gap persists in programs providing direct instruction on specific coping skills to directly target emotional distress or behavioral dysregulation.

Dialectical Behavior Therapy Skills Training for Emotional Problem Solving for Adolescents (DBT STEPS-A) is an SEL curriculum which allows general education teachers to teach mindfulness, emotion regulation, distress tolerance, and interpersonal effectiveness skills from dialectical behavior therapy (DBT) through 30 manualized lessons designed for 50 min periods (Mazza et al., 2016). DBT STEPS-A is based on the skills training component of DBT (Linehan, 1993), an evidence-based cognitive behavioral therapy that has repeatedly been shown to be effective for improving mental health outcomes in adolescents and adults (Chen et al., 2008; Chugani et al., 2013; Linehan et al., , 1999, 2006, 2015; Miller et al., 2006; Perepletchikova et al., 2011). Unlike therapies that target a specific symptom (e.g., depression), DBT has demonstrated transdiagnostic effectiveness (Neacsiu et al., 2014; Ritschel et al., 2015) for a wide range of mental health challenges including depression and anxiety (Panepinto et al., 2015; Ritschel et al., 2012), suicidality (Linehan et al., 2006, 2015), addiction (Dimeff \& Linehan, 2008; Linehan et al., 1999; Wilks et al., 2017), and eating disorders (Chen et al., 2008). Unlike many rigorously evaluated SEL programs, which show low or no efficacy for reducing emotional distress (Collaborative for Academic Social and Emotional Learning (CASEL), 2015), DBT STEPS-A directly targets mental health by combining mindfulness with skills for emotion regulation, distress tolerance, and interpersonal effectiveness and as such, was an ideal candidate for our pilot project, as our long term goal was to improve student mental health through reductions in distress and mental health symptoms (e.g., depression). The program shows preliminary effectiveness for reducing emotional symptoms and internalizing problems (e.g., depression, anxiety) in high school students (Flynn et al., 2018a, 2018b) and has also shown effectiveness for social resilience and difficulties with emotion regulation in racially diverse, rural ninth-graders (Martinez Jr. et al., 2021). However, the present study is the only investigation to date on the acceptability and feasibility of DBT STEPS-A and the only to focus on a low-income school. The focus on establishing 
acceptability and feasibility specifically with low-income schools is particularly important, as SEL programs like DBT STEPS-A will not likely reach these schools and students unless they are optimized to fit with available resources and to be culturally responsive to the needs and experiences of students. While larger, more rigorous trials of DBT STEPSA are needed to investigate efficacy, a first step in this area of inquiry is to establish the acceptability, appropriateness, and feasibility of the model to inform strategic adjustments prior to large-scale studies. In this study, we aimed to address this gap by: (1) evaluating an implementation of DBT STEPS-A integrated into required high school health classes, and (2) partnering with a low-income high school situated within a low-SES community to evaluate the potential for dissemination of the program into such communities.

\section{Background and Community Context}

The superintendent of a Southwestern Pennsylvania public school district assembled a coalition of community and school stakeholders to address a recently received designation for Additional Targeted Support and Improvement (A-TSI) by the Pennsylvania Department of Education due to racial disparities in academic achievement and school attendance (Pennsylvania Department of Education, 2019). Investigators from the University of University of Pittsburgh with expertise in school-based health initiatives were invited to attend. At this time, the superintendent expressed a desire to include an initiative to support the mental health of students in the district. Supporting student mental health within this district has been an ongoing challenge on several fronts. First, the district is located in a low-SES community with high rates of community violence. Within the high school, $67.3 \%$ of students are economically disadvantaged, $21.5 \%$ receive special education, and the racial demographics are as follows: $56.3 \%$ White, $34.3 \%$ Black, 1.8\% Hispanic, $1.1 \%$ Asian, and $6.4 \%$ two or more races. Within the school district overall, $27 \%$ of children live below the poverty line. Second, few mental health providers are available in the local community. Third, even when students are referred to the school's program for addressing mental health problems, parents often do not provide their consent for students to participate.

\section{Planning and Development}

Given the challenges in addressing student mental health outside of the school context, we devised a plan for bringing more mental health support into the school by implementing the DBT STEPS-A curriculum as an upstream approach to be integrated within high school health classes required for graduation. The standard training protocol for implementing DBT STEPS-A is attendance at a three-day training event led by the curriculum developers. Typically, school districts or collaborating community agencies contract with the program developers to offer the training, allowing teachers and behavioral health specialists from across the district to attend. It is also strongly recommended that a half-day training specifically geared toward school administrators be offered during initial training. Finally, it is recommended that schools implementing the program receive ongoing expert consultation during at least the first year of implementation at a rate of one hour per month.

When this ideal training scenario was presented to the district superintendent, it became clear that this would not be feasible for several reasons. First, the district would not be able to afford the cost of the training as it is a small district (two elementary, one middle, and one high school). Second, the time needed to send teachers to three consecutive days of training was not available. Third, the teacher's collective bargaining agreement mandates that any work they do outside of regular school hours (e.g., summer months) will result in additional hourly compensation. Fourth and perhaps most challenging to address, asking teachers to learn a completely new curriculum from a content area outside of their typical health class expertise was potentially overwhelming and could diminish teacher willingness and enthusiasm for the program. Instead of attempting the traditional training and implementation strategy for DBT STEPS-A, the superintendent recommended we explore the option of using a co-teaching model in which a fully trained individual would co-teach with high school health teachers, allowing them to learn the content and curriculum delivery over time. Given the clear barriers with the traditional training model and high need for mental health support in the school district, we agreed to develop a co-teaching model for program implementation.

The next challenge was to locate an appropriate coteacher to partner in the project. In our community, we are fortunate to have a community-based organization called the Homeless Children's Education Fund (HCEF), whose mission is to support the educational needs of children experiencing homelessness in Allegheny County. HCEF has found that it can often be most effective to reach these youth via schools, as they can be difficult to access outside of school hours due to parentification (having to take on a parent role for siblings), the need to work, housing instability, or difficulties accessing transportation. Three years ago, HCEF's teen program manager began offering college and career readiness programming in local public schools. Given that children experiencing homelessness have high rates of mental health symptoms and HCEF providers were already embedded within public school classrooms, it was a natural fit to invite HCEF's teen program manager to partner with us 
as the trained co-teacher. It is important to note that HCEF's teen program manager does not have any formal training or experience in the provision of mental health services, nor is such training required to teach DBT STEPS-A lessons. Though in this specific case, we leveraged a relationship with a community-based organization to partner with the school, we assert that the co-teaching model could be implemented with anyone meeting the following criteria:

1. Highly enthusiastic about bringing DBT STEPS-A to the local school district

2. Has access to classrooms and resources to do so (i.e., has time and ability to travel to school classrooms, able to pass all required background checks)

3. Willing to participate in a three-day DBT STEPS-A training

4. Committed to working as a co-teacher until health teachers are able to teach the lessons independently (we anticipate this process will take approximately 2 years)

\section{Methods}

\section{Training Protocol and Participants}

Our co-teaching model of implementation is based on the premise that when time and resources are scarce (such as in the case of many low-income schools), available support and resources should be directed most heavily onto key program implementers. We obtained an internal grant (Clinical and Translational Science Award) to support the project. The first step in this process was to send a community partner who agreed to serve as the co-teacher to a standard three-day DBT STEPS-A training event led by the program developers. Following this, one of the program developers provided a one-day presentation on DBT STEPS-A designed to enhance interest among key school stakeholders including 21 general education teachers, two special education teachers, two school counselors/psychologists, and three administrators. The one-day presentation was held during the summer months, and all teachers in attendance were compensated at their union-negotiated hourly rate hour for six training hours plus one hour for lunch. Lunch and a copy of the DBT STEPS-A manual were provided to all attendees.

\section{Program Launch}

In the high school, health class is a one-semester course, which meets five days per week, 42 minutes per class period. Thus, we decided (with input from one of the program developers) that DBT STEPS-A lessons would occur on Mondays and Thursdays. The curriculum delivery was structured this way for two primary reasons: (1) giving a few days between each lesson allows students some time to apply skills outside of the classroom in their lives before learning the next skill, and (2) it allows for some integration of skills training with other health content (e.g., discussion of how the skills apply to other health topics such as setting limits in an intimate relationship, having a discussion about consent, or saying no when offered drugs or alcohol). The high school health teachers $(n=2)$ considered their current health curriculum and found that they could dedicate 33 class periods to DBT STEPS-A while still adequately covering the remaining needed content to adhere to educational standards for high school health class. Though this would allow for delivery of the full 30-lesson curriculum, challenges related to length of class periods emerged early in the semester and it became necessary to switch to a 19-lesson version of the curriculum that would allow for DBT STEPS-A lessons to be delivered only once per week, with a few exceptions where lessons occurred twice per week. Time issues included in-class challenges such as needing to take more time than planned to explain certain concepts and the need to review homework assignments in class due to low completion rates, as well as logistical challenges that interfered with delivery of the program as originally planned (e.g., senior skip days or other school events that cut into health class periods). The team received expert consultation from one of the DBT STEPS-A program developers to address these challenges, including guidance on which parts of each lesson to shorten as well as recommendations on which lessons to completely omit. The 19-lesson curriculum was designed to cover key skills from all four core skills training modules and is consistent with other DBT skills training protocols adapted due to time constraints (Chugani et al., 2013). Figure 1 provides a flowchart of the overall implementation process (establishing partnerships, training, and program implementation).

\section{Role of Health Teachers and Co-Teacher}

Given that the health teachers had not received the standard 3-day training to deliver DBT STEPS-A, the co-teacher's role was to lead classes on days that program lessons were being delivered. Meanwhile, the health teachers added any additional information as needed, supported students in their work during these lessons, and related to content to other health topics taught on days when the co-teacher wasn't present (note that the co-teacher only joined health classes to teach DBT STEPS-A lessons twice per week). Since health teachers knew their students better than the co-teacher, they 
Fig. 1 Flowchart of partnerships, training, and implementation. PI $=$ Principal investigator




also found that it was easier for them to tell when a student needed something to be re-worded or explained differently to better grasp the content.

\section{Measures}

\section{Pre-Implementation Measures}

To assess how well the one-day presentation was received by high school teachers and administrators, an internally developed training evaluation was administered that included five quantitative items (e.g., "How valuable did you find today's training?") and four qualitative items (e.g., "What would have made this training more valuable/useful for you?"). The five quantitative items were investigator-developed items typically used in educational training events, and all were rated on a five-point Likert scale ranging from "not at all" (1) to "extremely" (5).

Participants in the one-day presentation also completed the Acceptability of Intervention Measure (AIM), Intervention Appropriateness Measure (IAM), and Feasibility of Intervention Measure (FIM), which are brief, validated scales of the acceptability, appropriateness, and feasibility of interventions (Weiner et al., 2017). Each scale has four items, and all three scales have demonstrated good discriminant and structural validity, test-retest reliability, and good internal consistency $(\alpha$ range $=0.85-0.91)$. Items are rated on a five-point Likert scale ranging from "completely disagree" (1) to "completely agree" (5).

\section{Post-Implementation Measures}

To examine fidelity and ease of use on a lesson-by-lesson basis, a brief, electronic fidelity form was created which the co-teacher completed after each lesson (one rating per class period). The fidelity form captures the rater, lesson number, class period number, whether the lesson content was fully delivered (if not, what percentage was delivered on a sliding scale of 0 to $100 \%$ ), how easy it was to teach the lesson (rated on a five-point Likert Scale from "extremely difficult" (1) to "extremely easy" (5)), how well the students seemed to like the lesson (rated on a five-point Likert Scale from "dislike a great deal" (1) to "like a great deal" (5)), and any other notes about the class including successes, challenges, or other interesting things that happened. In vivo or videorecorded fidelity ratings were outside of the scope of the current project.

Finally, the 29 teachers and administrators (including the health teachers and our trained co-teacher) who participated in the one-day presentation were invited to complete the AIM, IAM, and FIM at the end of the first semester during which the DBT STEPS-A was offered. In addition to these measures, four qualitative items gathered feedback on what else can be done to support program implementation, what participants would suggest if the implementation was done again, additional feedback about any part of the DBT STEPS-A curriculum or training, and feedback for the research team about how to improve the partnership with the school or district.

\section{Analyses}

Quantitative items were analyzed descriptively (mean, standard deviation, range). Qualitative data were transcribed electronically and uploaded to Dedoose, a secure online platform for qualitative data analysis (Sociocultural Research Consultants LLC, 2018). We set our benchmark for adequate acceptability, appropriateness, and feasibility as a mean rating of 4 (on a Likert scale of 1-5). Qualitative data were coded by a research assistant under the supervision of the first author using a descriptive qualitative coding method aiming to capture common patterns or themes in responses among participants (Sandelowski, 2000). The University of Pittsburgh Human Subjects Research Protections Office (HRPO) approved this study as an exempt protocol for evaluation of educational curricula.

\section{Results}

\section{Pre-Implementation Findings}

Overall, participants in the one-day presentation found the event to be valuable and agreed that DBT STEPS-A was important for high school students. Table 1 presents participants ratings on our internally developed training evaluation
Table 1 Participant ratings on training evaluation items

\begin{tabular}{llll}
\hline Item $(\mathrm{n}=29)$ & M & SD & Range \\
\hline 1. How valuable did you find today's training? & 3.93 & 0.75 & $2-5$ \\
2. How important do you think it is for high school students to learn STEPS-A skills? & 4.62 & 0.49 & $4-5$ \\
3. How important you do think it is to include skills for emotional well-being and & 4.59 & 0.69 & $2-5$ \\
problem solving in high school health curricula? & & & \\
4. How likely are you to use information you learned today in your teaching? & 3.93 & 0.70 & $2-5$ \\
5. How likely are you to use information you learned today in your personal life? & 3.90 & 0.77 & $2-5$ \\
\hline
\end{tabular}


Table 2 Descriptive Statistics by Item for the Feasibility of Intervention Measure (FIM)

\begin{tabular}{llll}
\hline Item $(\mathrm{n}=29)$ & $\mathrm{M}$ & $\mathrm{SD}$ & Range \\
\hline 1. DBT STEPS-A seems implementable & 4.17 & 0.60 & $3-5$ \\
2. DBT STEPS-A seems possible & 4.24 & 0.58 & $3-5$ \\
3. DBT STEPS-A seems doable & 4.17 & 0.54 & $3-5$ \\
4. DBT STEPS-A seems easy to use & 3.83 & 0.89 & $2-5$ \\
\hline
\end{tabular}

items $(n=29)$. Participant ratings also indicate that participants found DBT STEPS-A to be highly acceptable $(M=4.47, S D=0.57, \alpha=0.96)$ and appropriate $(M=4.55$, $S D=0.53, \alpha=0.94$ ) for their school and students. In comparison, the scores for feasibility of the program were lower, though the overall mean score remained supportive $(M=4.1$, $S D=0.57, \alpha=0.88)$. Given this, each individual feasibility item was examined and results revealed that "ease of use" as a particular area was perceived as a relative weakness (see Table 2). It is important to note that these ratings were provided prior to program implementation, at the end of the one-day presentation.

\section{Qualitative Feedback on the One-day Presentation}

Participants almost unanimously felt the topics covered during the one-day DBT STEPS-A presentation were important, needed, and valuable. Overall, well-being, coping strategies, and mental health were highlighted as areas where strategic improvements were wanted and necessary. Participants generally enjoyed the introduction to the DBT STEPS-A curriculum, though some also expressed interest in being able to see what the program was like once implemented. However, about one quarter of participants said they simply felt the presentation was too long or that there was too much information presented at once for them to feel comfortable putting it to use. This left some participants feeling overwhelmed after the presentation concluded.

Nearly all participants requested more information on how to implement the program and apply DBT STEPS-A techniques in real-life situations, with a few voicing logistical concerns. Among the suggestions related to what information would be desired should another training event occur were: (1) more time for training, (2) more engagement with trainees, and (3) more details on how to apply the program in their specific setting. Those who made these suggestions were interested in reviewing specific materials (e.g., lesson plans) and obtaining a more in-depth understanding of the skills and strategies than what was introduced. Most participants also suggested a more "hands on" approach, including a troubleshooting portion with time for questions and answers. Participants also requested the addition of case examples or role-play scenarios to enrich the learning experience and to better illustrate implementation.

\section{Post-Implementation Findings}

Acceptability, appropriateness, and feasibility were reassessed at the end of the first semester of implementation with the same 29 participants who attended the training event being invited to complete the post-semester evaluation. All original training participants were included in this evaluation regardless of their role in implementing the program with the focus on examining how the program was perceived by a variety of school stakeholders. Overall, 23 participants responded to the post-semester survey for a follow-up response rate of $79 \%$. Respondents included three health teachers (including the trained co-teacher), four high school/district administrators, 15 general education teachers not directly involved in teaching DBT STEPS-A lessons as part of health class, and one respondent who did not indicate their job role. Table 3 presents post-implementation ratings of program acceptability, appropriateness, and feasibility for the overall group of respondents, health teachers/co-teacher

Table 3 Post-Implementation Ratings of Acceptability, Appropriateness, and Feasibility Overall, Among Those who Delivered DBT STEPS-A Lessons, and Among Teachers, Staff, and Administrators

\begin{tabular}{llll}
\hline Construct & M & SD & Range \\
\hline Acceptability $(\mathrm{n}=23)$ & 3.87 & 0.57 & $2.75-4.75$ \\
Health Teachers/Co-Teacher $(\mathrm{n}=3)$ & 4.33 & 0.14 & $4.25-4.5$ \\
Teachers/Staff/Administrators $(\mathrm{n}=19)$ & 3.84 & 0.56 & $2.75-4.75$ \\
Appropriateness $(\mathrm{n}=23)$ & 3.95 & 0.62 & $3-5$ \\
Health Teachers/Co-Teacher $(\mathrm{n}=3)$ & 3.75 & 0.66 & $3-4.25$ \\
Teachers/Staff/Administrators $(\mathrm{n}=19)$ & 4.03 & 0.60 & $3-5$ \\
Feasibility $(\mathrm{n}=21)$ & 3.74 & 0.65 & $2.25-5$ \\
Health Teachers/Co-Teacher $(\mathrm{n}=3)$ & 4.25 & 0.50 & $3.75-4.75$ \\
Teachers/Staff/Administrators $(\mathrm{n}=17)$ & 3.69 & 0.63 & $2.25-5$ \\
\hline
\end{tabular}

Table 4 Post-Implementation Feasibility Ratings by Item Among Those Who Delivered DBT STEPS-A Lessons (Health Teachers and Co-Teacher; $n=3$ )

\begin{tabular}{llll}
\hline Item & $\mathrm{M}$ & $\mathrm{SD}$ & Range \\
\hline 1. DBT STEPS-A seems implementable & 4.33 & 0.58 & $4-5$ \\
2. DBT STEPS-A seems possible & 4.33 & 0.58 & $4-5$ \\
3. DBT STEPS-A seems doable & 4.33 & 0.58 & $4-5$ \\
4. DBT STEPS-A seems easy to use & 4 & 1 & $3-5$ \\
\hline
\end{tabular}


alone (i.e., those who actually delivered the program), and teachers, school staff, and administrators who were trained during the summer but do not have direct responsibility for program delivery. The overall mean scores were 3.87 for program acceptability (4.33 among those who delivered the program), 3.95 for program appropriateness (3.75 among those who delivered the program), and 3.74 for feasibility (4.25 among those who delivered the program). Table 4 presents the mean ratings for program feasibility by item among health teachers and the co-teacher $(n=3$; i.e., those who directly delivered the program).

\section{Qualitative Program Feedback}

Of the 23 follow-up survey participants, 12 provided qualitative feedback about the program through four open-ended questions. Overall, respondents shared a desire for more time to implement the program and more post-training collaboration and contact with the trainer, which if available, would have improved the programming experience. While there was no negative feedback about DBT STEPS-A as a program, participants who did not have direct involvement with teaching DBT STEPS-A lessons lacked understanding of how to support students in using the skills, though they expressed a strong desire to be able to do so. Some participants also expressed a desire to be evaluated, reporting that they would like to know how well they are doing in implementing the program.

When asked about what changes they would like to see, participants were concerned with logistical and administrative details, such as a desire for more communication between those who did vs. did not have direct responsibility for delivering the program, limited accessibility to the program for all students (e.g., lack of opportunity for those who have already taken health class prior to implementation) and tailoring teaching strategies based on student needs (e.g., age-based content for younger vs. older students, material for at-risk students, etc.). Overall, participant responses were less focused on changing the content of the program itself as they were on increasing the level of support and implementation adaptation for their setting and student needs, including a desire for follow-up training and consistent, situational guidance from their trainer(s).

Among the three participants who had direct involvement in teaching DBT STEPS-A lessons, two provided qualitative feedback. The responses indicate that they felt poorly prepared to implement the program, wanted to consult with other teachers delivering the program, and desired a better understanding of the practical application of the skills. They also emphasized needing more training overall, continued support, and opportunities for personalized consultation.

\section{Fidelity Ratings}

The co-teacher, who received the standard three-day training in DBT STEPS-A, provided fidelity ratings throughout the first semester of implementation. Of the 69 DBT STEPS-A classes taught during the fall 2019 semester (23 lessons per class section with 3 sections total), teachers were able to deliver the full lesson content $71 \%$ of the time. When lesson content was not fully delivered, the co-teacher estimated that the amount of content that was delivered ranged from 15 to $94 \%$. The average rating for ease of use for the lessons was $3.30(S D=0.086)$. The average rating for how well students seemed to like the lesson was $3.52(S D=0.093)$. Thus, while the overall ratings for ease of use of the program indicate an acceptable degree of program feasibility (see Table 4), these lesson-by-lesson ratings indicate that ease of use of certain lessons and student responsiveness to specific lessons could be improved.

\section{Discussion}

The data presented here are the first to explore acceptability, appropriateness, and feasibility of the DBT STEPS-A program in a low-income school, as well as to present a detailed account of program implementation and on-theground adaptations. While DBT STEPS-A was rated as adequately acceptable, appropriate, and feasible by school stakeholders immediately after training and prior to implementation, these ratings dropped below the benchmark of an average rating of 4 post-implementation. Additionally, these ratings varied among those who did vs. did not directly deliver DBT STEPS-A lessons. Among those with direct involvement in teaching the program, acceptability and feasibility were adequate, while appropriateness fell below the benchmark. Interestingly, while program ease of use was adequate among those with direct involvement, lesson-bylesson ratings for ease of use (provided by the co-teacher, who received the standard 3-day training to deliver DBT STEPS-A) fell well below the benchmark. One explanation for this lower rating may be due to the fact that this was the first time the co-teacher had implemented the DBT STEPSA curriculum and that ease of use may increase with greater familiarity. In addition, although the co-teacher had experience providing programming in high schools, he is not a high school teacher, and this may have impacted ease of use of the program. Post-implementation qualitative feedback further indicated that school stakeholders had questions about how to support students in using DBT STEPS-A skills outside of health class, how students who had already taken health could be exposed to the program, and a desire for more ongoing training and contact with trainers. Additionally, it is important to note that the implementation at 
this school represents a substantial departure from the recommended training protocols, which were not financially feasible for this school district. Given that the training provided to this group of stakeholders was so limited, it is not surprising that some felt ill-prepared to use the program. Additional grant funding to support the cost of the standard 3-day DBT STEPS-A training for the health teachers as well as dedicated financial resources (e.g., substitute teacher coverage) to protect health teachers' time to participate in training and prepare to deliver DBT STEPS-A lessons may have led to better confidence for teachers tasked with learning and delivering the program. Interestingly, the two health teachers, neither of whom received the standard three-day training to deliver the program, rated it as both adequately acceptable and feasible. Further, while the stakeholder ratings overall fell below our a priori benchmark of 4 , the ratings were just slightly below the benchmark. Given that this implementation took place in a low-income school, it is possible that the program may not fully attend to the needs of students living in areas with high rates of poverty and community violence or may need further adaptation to meet the needs of a racially and ethnically diverse student population. Further program refinement based on more in-depth qualitative interviews, including student feedback, would likely be of substantial benefit in informing next steps, especially as the curriculum adaptations did not directly assess cultural responsiveness or mismatch in the present study.

Several major challenges in implementation were also observed during the first semester of implementation, and these were largely consistent with barriers to implementation of other school-based programs (Langley et al., 2010). First, health teachers were extremely busy and there were no opportunities other than in-service days for them to meet with the trained co-teacher to discuss progress and planning for transitioning them toward independent delivery of the curriculum. In essence, though our data indicate that more training, contact with trainers, and personal consultation was desired, the reality was that there was little time during which this could occur. While the co-teacher received monthly expert consultation throughout the semester (hourlong phone calls) and emailed for further assistance as needed, the health teachers were unable to participate in this support because they did not have time to participate during their workday and could not participate outside of their workday. This situation was partially remedied by arranging for the health teachers and co-teachers to meet once weekly during their early morning planning period to review the contents of each lesson prior to delivering the lesson in class later the same day. To address the issue of supporting health teachers in their transition to teaching DBT STEPSA independently, the research team, co-teacher, and health teachers met and made a plan for gradual transition over the following three semesters including first teaching the lesson introduction, then teaching about half the lesson, and then teaching the lesson independently with the co-teacher observing and supporting as needed. This transition plan was developed in consultation with the health teachers, who agreed that the timing of the plan was reasonable and that they were comfortable with the program material, although no additional learning opportunities beyond the 1-day training related to DBT STEPS-A were provided.

The co-teacher also found that it was difficult to adequately deliver the DBT STEPS-A lessons as written given the lessons are designed for $50 \mathrm{~min}$ and class periods in this high school are $42 \mathrm{~min}$. While the co-teacher received expert consultation from the program developers about where to trim down lesson content, they found that this was not sufficient and decided that it would be more feasible to switch to a 19-lesson version of the curriculum which was developed in consultation with one of the DBT STEPS-A program developers and the health teachers. Finally, students did not complete their assigned DBT STEPS-A homework as they were unaccustomed to receiving homework in health class. The co-teacher found that the most effective way to handle this was to encourage students to complete the homework sheets during the next lesson review, as well as to start completing them during the end of each class period. For the following semester, health teachers agreed to make DBT STEPS-A homework completion a graded journal entry that all students would complete during the day after the DBT STEPS-A lesson was delivered. Health teachers also agreed to facilitate one additional mindfulness activity per week on a non-DBT STEPS-A lesson day to further integrate these skills into the overall health curriculum and reinforce the materials.

\section{Limitations}

This study is limited by a small sample within a single high school. The data are limited to pre- and post-implementation ratings of intervention acceptability, appropriateness, feasibility, qualitative feedback, and basic lesson fidelity ratings, and do not include preliminary effectiveness data. An important limitation of the present work is that it does not include direct feedback from students, which would aid in strategic adaptations for cultural responsiveness and relevance. A second limitation is that the fidelity ratings reported here are self-reported, as a validated fidelity measure for DBT STEPS-A is not available and neither in vivo nor recorded observations were possible for the present study. Constructs such as acceptability, appropriateness, and feasibility may vary according to quality of implementation, and as such, our findings should be interpreted with this limitation in mind. Despite these limitations, this pilot study is the first to explore key implementation variables in the delivery of DBT 
STEPS-A and to focus specifically on collaborating with school stakeholders to adapt programs to enhance feasibility for low-resource schools. This pilot work has revealed that despite many implementation challenges, DBT STEPS-A is acceptable to those who implement the program and that future work should focus on improving the feasibility and appropriateness of the program as well as culturally responsive adaptations.

\section{Future Directions}

Although DBT STEPS-A has been implemented in other low-income schools in the USA, no research to date has documented the extent to which this SEL program is acceptable and culturally responsive to the needs of students in low-income schools; these areas, along with feasibility for implementation in low-income schools, should be prioritized in future research on DBT STEPS-A. Our research plan for this project was to first address immediate issues related to implementation during the first semester of program implementation (as reported in this article) and next, to study the preliminary effectiveness and student perceptions of program acceptability during subsequent semesters of implementation. Unfortunately, the second semester of our program implementation coincided with the COVID-19 lockdown beginning in March 2020. With the school closed, it became clear that teachers needed to focus on core curricula, and we were unable to continue this research. To adapt to these challenges, HCEF developed a virtual, after-school program in which students experiencing homelessness could receive DBT STEPS-A lessons via Zoom groups and we are now working toward evaluating this program. At the time of this writing, schools are beginning to re-open and we hope to resume our efforts to offer and investigate DBT STEPS-A in the high school as soon as it is feasible. The effects of the COVID-19 pandemic on the mental health of adolescents are already documented (Singh et al., 2020). Future research should address the impact that mental health focused SEL programs, like DBT STEPS-A, can have in supporting young people through the aftermath of the pandemic and in their transition to adulthood, college, and the workforce.

Funding This research is supported by a grant from the University of Pittsburgh Clinical and Translational Science Institute (CTSI; PI: Chugani).

\section{Declaration}

Conflicts of Interest Dr. Mazza is an author of "Dialectical Behavior Therapy Skills Training for Emotional Problem Solving for Adolescents (DBT STEPS-A)" and receives royalties, honoraria, and consult- ing fees related to its use. Dr. Chugani receives consulting fees from the Citrone 33 Foundation.

\section{References}

Chen, E., Matthews, L., Allen, C., Kuo, J., \& Linehan, M. (2008). Dialectical behavior therapy for clients with binge eating disorder or bulimia nervosa and borderline personality disorder. International Journal of Eating Disorders, 41(6), 505-512.

Chugani, C. D., Ghali, M. N., \& Brunner, J. (2013). Effectiveness of short term dialectical behavior therapy skills training in college students with cluster b personality disorders. Journal of College Student Psychotherapy, 27(4), 323-336.

Coley, R., Sims, J., Dearing, E., \& Spielvogel, B. (2018). Locating economic risks for adolescent mental and behavioral health: Poverty and affluence in families, neighborhoods, and schools. Child Development, 89(2), 360-369.

Collaborative for Academic Social and Emotional Learning (CASEL). (2015). 2015 CASEL Guide: Effective social and emotional learning programs. http://secondaryguide.casel.org/casel-secondaryguide.pdf

Deighton, J., Lereya, S., Casey, P., \& Patalay, P. (2019). Prevalence of mental health problems in schools: poverty and other risk factors among 28,000 adolescents in England. The British Journal of Psychiatry. https://doi.org/10.1192/bjp.2019.19

Dimeff, L., \& Linehan, M. (2008). Dialectical behavior therapy for substance abusers. Addiction Science \& Clinical Practice, 4(2), 39-47.

Durlak, J., Weissberg, R., Dymnicki, A., Taylor, R., \& Schellinger, K. (2011). The impact of enhancing students' social and emotional learning: A meta-analysis of school-based universal interventions. Child Development, 82(1), 405-423.

Flynn, D., Joyce, M., Weihrauch, M., \& Corcoran, P. (2018a). Innovations in practice: Dialectical behaviour therapy - skills training for emotional problem solving for adolescents (DBT STEPSA): Evaluation of a pilot implementation in Irish post-primary schools. Child and Adolescent Mental Health, 23(4), 376-380.

Flynn, D., Joyce, M., Weihrauch, M., Corcoran, P., Gallagher, E., O'Sullivan, C., \& Hurley, P. (2018). Dialectical behaviour therapy - Skills training for emotional problem solving for adolescents (DBT STEPS-A): Pilot implementation in an Irish context. https:// www.hse.ie/eng/services/list/4/mental-health-services/dbt/proj/ res/dbt-steps-a-report-on-the-pilot-implementation-in-ireland.pdf

Langley, A., Nadeem, E., Kataoka, S., Stein, B., \& Jaycox, L. (2010). Evidence-based mental health programs in schools: Barriers and facilitators of successful implementation. School Mental Health, 2, 105-113.

Linehan, M. M. (1993). Cognitive behavioral treatment of borderline personality disorder. Guildford Press.

Linehan, M. M., Comtois, K. A., Murray, A. M., Brown, M. Z., Gallop, R. J., Heard, H. L., Korslund, K. E., Tutek, D. A., Reynolds, S. A., \& Lindenboim, N. (2006). Two-year randomized controlled trial and follow-up of dialectical behavior therapy vs therapy by experts for suicidal behaviors and borderline personality disorder. Archives of General Psychiatry, 63(7), 757-766.

Linehan, M. M., Korslund, K. E., Harned, M., Gallop, R., Lungu, A., Neacsiu, A., McDavid, J., Comtois, K. A., \& Murray-Gregory, A. (2015). Dialectical behavior therapy for high suicide risk in individuals with borderline personality disorder: A randomized clinical trial and component analysis. JAMA Psychiatry, 72(5), 475-482.

Linehan, M. M., Schmidt, H., III., Dimeff, L. A., Craft, J. C., Kanter, J., \& Comtois, K. A. (1999). Dialectical behavior therapy for patient 
with borderline personality disorder and drug-dependence. The American Journal on Addictions, 8(4), 279-292.

LoMurray, M. (2005). Sources of Strength Facilitators Guide: Suicide prevention gatekeeper training. The North Dakota Suicide Prevention Project.

Low, S., Smolkowski, K., \& Cook, C. (2016). What constitutes highquality implementation of SEL program? A latent class analysis of Second Step implementation. Prevention Science, 17, 981-991.

Martinez Jr., R. R., Marraccini, M. E., Knotek, S. E., Neshkes, R. A., \& Vanderburg, J. (2021). Effects of dialectical behavior therapy skills training for emotional problem solving for adolescents (DBT STEPS-A) program of rural ninth-grade students. School Mental Health, 1-14. https://doi.org/10.1007/s12310-021-09463-5

Mazza, J., Dexter-Mazza, E., Miller, A., Rathus, J., \& Murphy, H. (2016). DBT skills in schools: Skills training for emotional problem solving for adolescents (DBT STEPS-A). New York: Guilford Press.

Miller, A., Rathus, J., \& Linehan, M. M. (2006). Dialectical behavior therapy with suicidal adolescents. Guilford Press.

MindWise Innovations. (2021). The SOS Signs of Suicide Program. Retrieved April 14 from https://www.mindwise.org/suicide-preve ntion/

National Academies of Sciences, E., and Medicine. (2019). Fostering healthy mental, emotional, and behavioral development in children and youth: A national agenda. The National Academies Press. https://doi.org/10.17226/25201

Neacsiu, A., Eberle, J., Kramer, R., Wiesmann, T., \& Linehan, M. (2014). Dialectical behavior therapy skills for transdiagnostic emotion dysregulation: A pilot randomized controlled trial. Behaviour Research and Therapy, 59, 40-51.

Panepinto, A., Uschold, C., Olandese, M., \& Linn, B. (2015). Beyond borderline personality disorder: dialectical behavior therapy in a college counseling center. Journal of College Student Psychotherapy, 29(3), 211-226.

Pennsylvania Department of Education. (2019). School improvement and accountability. Retrieved April 18 from https://www.educa tion.pa.gov/K-12/ESSA/Pages/Accountability.aspx

Perepletchikova, F., Axelrod, S., Kaufman, J., Rounsaville, B., Douglas-Palumberi, H., \& Miller, A. (2011). Adapting dialectical behaviour therapy for children: Towards a new research agenda for pediatric suicidal and non-suicidal self-injurious behaviours. Children and Adolescent Mental Health, 16(2), 116-121.

Ritschel, L., Cheavens, J., \& Nelson, J. (2012). Dialectical behavior therapy in an intensive outpatient program with a mixed-diagnostic sample. Journal of Clinical Psychology, 68(3), 221-235.

Ritschel, L., Lim, N., \& Stewart, L. (2015). Transdiagnostic applications of DBT for adolescents and adults. American Journal of Psychotherapy, 69(2), 111-128.

Sandelowski, M. (2000). Whatever happened to qualitative description? Research in Nursing \& Health, 22, 334-340.
Schilling, E., Aseltine, R., \& James, A. (2016). The SOS suicide prevention program: Further evidence of efficacy and effectiveness. Prevention Science, 17, 157-166. https://doi.org/10.1007/ s11121-015-0594-3

Shim, R., Kho, C., \& Murray-Garcia, J. (2018). Inequities in mental health and mental health care: A review and future directions. Psychiatric Annals, 48(3), 138-142.

Silberholz, E., Brodie, N., Spector, N., \& Pattishall, A. (2017). Disparities in access to care in marginalized populations. Current Opinion in Pediatrics, 29(6), 718-727.

Singh, S., Roy, D., Sinha, K., Parveen, S., Sharma, G., \& Joshi, G. (2020). Impact of COVID-19 and lockdown on mental health of children and adolescents: A narrative review with recommendations. Psychiatry Research, 293, 1-11.

Sklad, M., Diekstra, R., De Ritter, M., Ben, J., \& Gravesteijn, C. (2012). Effectiveness of school-based universal social, emotional, and behavior programs: Do they enhance students' development in the area of skills, behavior, and adjustment? Psychology in the Schools, 49(9), 892-909.

Sociocultural Research Consultants LLC. (2018). Dedoose Version 8.0.35, web application for managing, analyzing, and presenting qualitative and mixed method research data. In www.dedoo se.com

Taylor, R., Oberle, E., Durlak, J., \& Weissberg, R. (2017). Promotion positive youth development through school-based social and emotional learning interventions: A meta-analysis of follow-up effects. Child Development, 88(4), 1156-1171.

Weiner, B., Lewis, C., Stanick, C., Powell, B., Dorsey, C., Clary, A., Boynton, M., \& Halko, H. (2017). Psychometric assessment of three newly developed implementation outcome measures. Implementation Science, 12(108), 1-12.

Wilks, C., Ang, S., Matsumiya, B., Lungu, A., \& Linehan, M. (2017). Internet-delivered dialectical behavioral therapy skills training for suicidal and heavy episodic drinkers: Protocol and preliminary results of a randomized controlled trial. JMIR Research Protocols, 25(6), e207.

World Health Organization and Calouste Gulbenkian Foundation. (2014). Social determinants of mental health. https://apps.who. int/iris/bitstream/handle/10665/112828/9789241506809_eng. pdf;jsessionid=92963865D0845F1DBD446D1D7D312450? sequence $=1$

Wyman, P., Brown, C., LoMurray, M., Schmeelk-Cone, K., Petrova, M., Yu, Q., Walsh, E., Tu, X., \& Wang, W. (2010). An outcome evaluation of the Sources of Strength suicide prevention program delivered by adolescent peer leaders in high schools. American Journal of Public Health, 100(9), 1653-1661. https://doi.org/10. 2105/AJPH.2009.190025

Publisher's Note Springer Nature remains neutral with regard to jurisdictional claims in published maps and institutional affiliations. 